\title{
LA DRAMATIZACIÓN COMO HERRAMIENTA DIDÁCTICA PARA LA MEJORA DE LA CALIDAD DEL APRENDIZAJE EN LA EXPRESIÓN ORAL DEL IDIOMA INGLÉS, NIVEL INICIAL INSTITUCIÓN EDUCATIVA PRIVADA CANONESAS DE LA CRUZ - PUEBLO LIBRE, LIMA 2018
}

\author{
Brenda Patricia SIMÓN CHAHUILLCO ${ }^{1}$ \\ Ofelia Carmen SANTOS JIMÉNEZ ${ }^{2}$ \\ https://orcid.org/0000-0003-1294-0641
}

\begin{tabular}{|lll|}
\hline Recibido & $:$ & 20.03 .2020 \\
Aceptado & $:$ & 15.06 .2020 \\
Publicado & $:$ & 06.07 .2020 \\
\hline
\end{tabular}

\begin{abstract}
RESUMEN: El propósito de la investigación fue aplicar la dramatización como herramienta didáctica para la mejora la calidad de la expresión oral del idioma inglés en los niños y niñas de 5 años de la institución Educativa Privada Canonesas De la Cruz-Pueblo Libre, Lima-2018. El tipo de investigación fue aplicada, el método utilizado en la investigación fue el método científico. El diseño fue el cuasi experimental con dos grupos equivalentes. La muestra estuvo conformada por 25 inglés en los niños y niñas de 5 años; la técnica de recolección de datos fue la aplicación de la dramatización para ambos grupos una Prueba pre test y Prueba Post test con su instrumento la lista de cotejo, Ficha de Observación de Sesiones de Aprendizajes, con validez y confiabilidad. Se realizaron una serie de unidades didácticas utilizando la dramatización para los dos grupos. La hipótesis fue probada utilizando el estadístico de la $\mathrm{T}$ de Student para muestras independientes, mediante el programa SPSS versión 25. Las conclusiones que se aterrizó, la aplicación de la dramatización ayuda a los niños y niñas a experimentar sin miedo y a conseguir una adecuada expresión oral en el idioma inglés, con 5\% de margen de error, con P-valor $=0$ menor al 0,05 de significancia. La media aritmética en el Post Test para el grupo control fue de 51.5 y para el grupo experimental alcanzó 75.2, con una diferencia significativa para ambas variables.
\end{abstract}

Palabras claves: dramatización, didáctica, expresión oral, inglés.

Dramatization as a didactic tool for the improvement of the quality of learning in the oral expression of the english language, initial level of the Institución Educativa Privada Canonesas de La Cruz - Pueblo Libre, Lima 2018

\begin{abstract}
The purpose of the research was to apply dramatization as a didactic tool to improve the quality of oral expression of the English language in 5-year-old boys and girls from the Canonesas De la Cruz-Pueblo Libre Educational Institution, Lima-2018. The type of research was applied, the method used in the research was the scientific method. The design was quasi-experimental with two equivalent groups. The sample consisted of 25 English in 5-year-old boys and girls; The data collection technique was the application of dramatization for both groups, a pre-test and post-test with their instrument, the checklist, observation form of learning sessions, with validity and reliability. A series of didactic units were carried out using dramatization for the two groups. The hypothesis was tested using the Student $T$ statistic for independent samples, using the SPSS version 25 program. The conclusions that were landed, the application of dramatization helps children to experiment without fear and to achieve an adequate oral expression in the English language, with a 5\% margin of error, with P-value $=0$ less than 0,05 of significance. The arithmetic mean in the Post Test for the control group was 51.5 and for the experimental group it was 75.2, with a significant difference for both variables.
\end{abstract}

Keywords: dramatization, didactic, oral expression, English.

\footnotetext{
${ }^{1}$ Universidad Nacional Mayor de San Marcos. Email: brendapatriciasimon@gmail.com

${ }^{2}$ Universidad Nacional Mayor de San Marcos. Email: ofelia.santos@unmsm.edu.pe
} 


\section{Journal of the Academy $|120|$}

\section{INTRODUCCIÓN}

En esta era de constantes avances tecnológicos, científicos, culturales y sociales; el inglés ha llegado a tener una gran importancia a nivel mundial, permitiendo a los países abrir sus fronteras al intercambio global.

Es por esa razón que el idioma inglés es considerado, el idioma universal por ser el tercer idioma más hablado en el mundo. Hoy más que nunca resulta importante aprender este idioma; pues es una herramienta útil que permite el acceso a información; además que cada día se emplea en casi todas las áreas de conocimiento y desarrollo humano. Según el Diseño Curricular (2016).

El inglés permite adquirir información de los últimos avances científicos y tecnológicos, así también como permite el acceso a nuevas tecnologías de la información y la comunicación para ampliar el horizonte cultural".

Lo mencionado anteriormente en el diseño curricular involucra el aprendizaje del idioma inglés y la importancia que este tiene para el aprendizaje de todas las personas, incluyendo a los más pequeños que como sabemos tienen la ventaja de aprender con mayor facilidad un segundo idioma porque se les va inculcando algunas capacidades que pueden desarrollar desde niños, los cuales son: el saber escuchar, hablar y pronunciar las nuevas palabras enseñadas en el segundo idioma.

Ahora nos preguntamos ¿Por qué enseñar inglés a niños pequeños? Sabemos que los niños aceptan con facilidad un nuevo código lingüístico porque aún están aprendiendo el suyo. Les resulta fácil pronunciar los sonidos nuevos por el mismo motivo. No sienten vergüenza al hablar de una forma diferente. Si tienen una buena experiencia al aprender un idioma en esta edad, lo recordarán con cariño y así les será más fácil aprender otro idioma más tarde o mejorar el ya aprendido.

Muchos de los objetivos educativos a largo plazo al enseñar idiomas a niños pequeños, como sabemos es: preparar al niño para entender y aceptar diferentes modos de pensar y aprender y así enseñarle a ser libre de prejuicios. Ofrecer otro instrumento para organizar el conocimiento ayudando al desarrollo cognitivo del niño. Fomentar más creatividad como consecuencia de la comparación constante entre dos códigos lingüísticos.

Poner los cimientos para una educación lingüística continua. Brindar una formación que no se centra exclusivamente en la lengua materna de uno y que ayuda al estudio de lenguas extranjeras en edades posteriores.

Viendo la importancia que involucra el aprendizaje del idioma inglés en los niños de inicial, podemos mencionar que no sólo se debe contribuir al aprendizaje de los niños de una forma tradicional en donde los profesores solo se dedican a ejecutar por decirlo así su plan o clase y no muestran a la hora de enseñar la parte dinámica en donde ellos se pueden valer de muchos recursos educativos didácticos lo cual contribuya en el aprendizaje de los niños.

Teniendo en cuenta nuestras experiencias de enseñanza de aprendizaje en el nivel de inicial al momento que hicimos nuestras prácticas vimos la triste realidad de que el aprendizaje por parte de los niños no era el mejor en cuanto al aprendizaje del idioma inglés, Se pudo observar que los docentes no hacían uso de las diferentes estrategias didácticas que existen como es usar en sus sesiones de clase el uso de canciones, juegos de roles, el empleo de la dramatización para desarrollar sus habilidades y sentirse en un buen ambiente que se muestre presto a su aprendizaje.

Se puede observar que los niños eran muy cohibidos, no expresaban su ánimo al aprender, solo se dedicaban a ser pasivos más no activos. Frente a esta realidad es necesario asumir el reto de plantear una metodología didáctica diferente que asegure la enseñanza del Idioma Inglés; utilizando 


\section{Journal of the Academy $|121|$}

principalmente el empleo de la dramatización como estrategia metodológica que contribuya en el aprendizaje del idioma inglés.

\section{DESARROLLO}

\section{Objetivo general}

Aplicar la dramatización como herramienta didáctica para la mejora de la expresión oral del idioma inglés en los niños y niñas de 5 años de la institución Educativa Privada Canonesas De la Cruz-Pueblo Libre, Lima-2018.

\section{Metodología}

\section{Tipo de investigación}

- La presente investigación es de tipo aplicada, porque está encaminada hacia la solución del problema reconocido y programado, logrando respuestas cuantitativas.

- Es cuasi experimental porque ha realizado la manipulación de la variable independiente, para ver los resultados y la medición de la variable dependiente, con dos grupos uno experimental y el otro de control

- Tiene un alcance de nivel explicativo, ya que ha permitido que los resultados obtenidos demostraron el nivel de causa efecto de la variable independiente hacia la variable dependiente.

\begin{tabular}{|l|l|l|l|}
\hline $\begin{array}{l}\text { GRUPO EXPERIMENTAL } \\
\text { (casual) } \\
\text { Ge I }\end{array}$ & $\begin{array}{l}\text { PRETEST } \\
\mathrm{O}_{1}\end{array}$ & $\begin{array}{l}\text { TRATAMIENTO } \\
\text { X }\end{array}$ & $\begin{array}{l}\text { POSTEST } \\
\mathrm{O}_{2}\end{array}$ \\
\hline $\begin{array}{l}\text { GRUPO CONTROL } \\
\text { (casual) } \\
\text { Gc I }\end{array}$ & $\begin{array}{l}\text { PRETEST } \\
\mathbf{O}_{1}\end{array}$ & & $\begin{array}{l}\text { POSTEST } \\
\mathbf{O}_{2}\end{array}$ \\
\hline
\end{tabular}

Tabla 1

Diagrama de diseño experimental

\section{Discusión de resultados}

Se comienza la discusión de los resultados de acuerdo al objetivo de la investigación que provenía del siguiente problema ¿Cómo la dramatización como herramienta didáctica mejora la calidad de la expresión oral del idioma inglés en los niños y niñas de 5 años de la institución Educativa Privada Canonesas De la Cruz-Pueblo Libre, Lima-2018? De acuerdo a los pasos del método científico, fue necesario revisar otros trabajos de investigación con el fin de conocer quienes estudiaron estas variables de estudio, donde se halló antecedentes de investigación tanto en el ámbito internacional y nacional, de aquellos trabajos de investigación que abordaron la variable de estudio sobre la dramatización como herramienta didáctica mejora significativamente la calidad de la expresión oral del idioma inglés en los niños y niñas de 5 años.

Aplicado los instrumentos de investigación, recolectado los datos y analizado cada dimensión, nos proyectaron resultados descriptivos que responde al objetivo de la investigación en la siguiente manera. 
Resultados del pre-test

Cotejo Pre-Test Grupo Experimental

Variable: Calidad de la expresión oral del idioma inglés

DIMENSIÓN: DICCIÓN

\begin{tabular}{llllll} 
& & Frecuencia & Porcentaje & Porcentaje válido $\begin{array}{l}\text { Porcentaje } \\
\text { acumulado }\end{array}$ \\
\hline \multirow{3}{*}{ Válido } & NO & 9 & 69,23 & 69,23 & 69,23 \\
\cline { 2 - 6 } & SI & 4 & 30,77 & 30,77 & 100,0 \\
\cline { 2 - 6 } & Total & 13 & 100,0 & 100,0 & \\
\hline
\end{tabular}

Figura 01

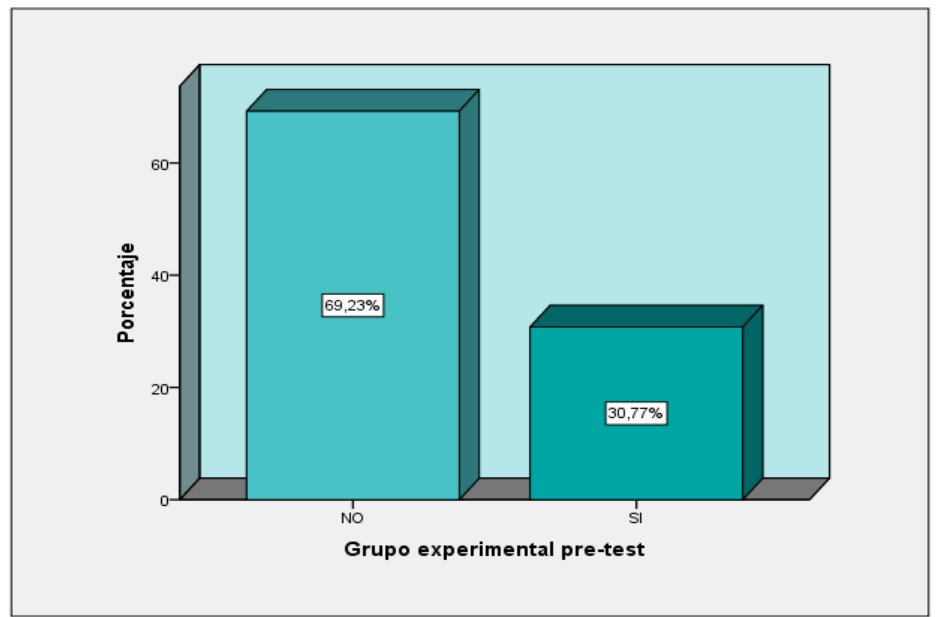

DIMENSIÓN: FLUIDEZ

Cuadro 12

\begin{tabular}{lllllll} 
& & Frecuencia & Porcentaje & Porcentaje válido & $\begin{array}{l}\text { Porcentaje } \\
\text { acumulado }\end{array}$ \\
\hline \multirow{3}{*}{ Válido } & NO & 6 & 46,15 & 46,15 & 46,15 \\
\cline { 2 - 6 } & SI & 7 & 53,85 & 53,85 & 100,0 \\
\cline { 2 - 6 } & Total & 13 & 100,0 & 100,0 & \\
\hline
\end{tabular}

Figura 02

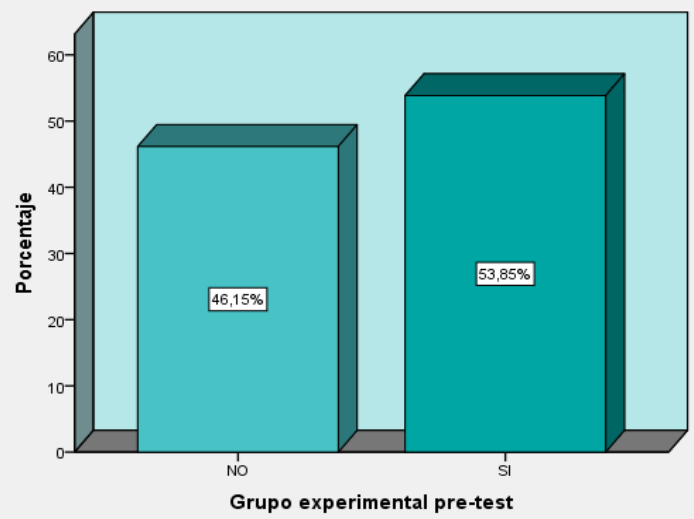


DIMENSIÓN: CLARIDAD

Cuadro 13

\begin{tabular}{lll|lll} 
& & Frecuencia & Porcentaje & $\begin{array}{l}\text { Porcentaje } \\
\text { válido }\end{array}$ & $\begin{array}{l}\text { Porcentaje } \\
\text { acumulado }\end{array}$ \\
\hline \multirow{2}{*}{ Válido } & NO & 9 & 69,23 & 69,23 & 69,23 \\
\cline { 2 - 6 } & SI & 4 & 30,77 & 30,77 & 100,0 \\
\cline { 2 - 6 } & Total & 13 & 100,0 & 100,0 & \\
\hline
\end{tabular}

Figura 03

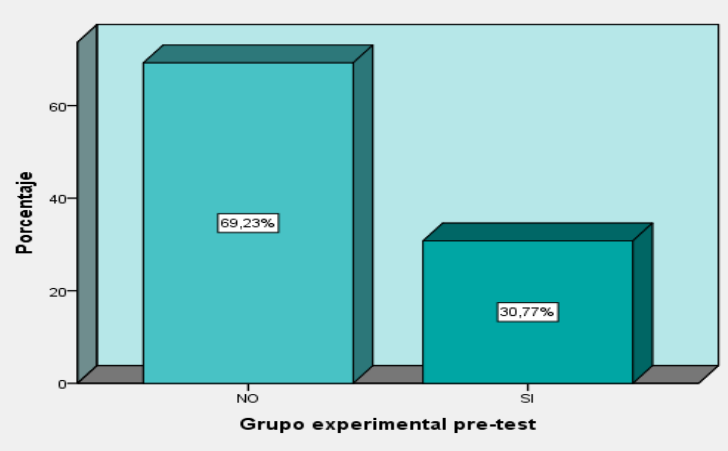

DIMENSIÓN: RITMO

\section{Cuadro 14}

\begin{tabular}{llllll} 
& & Frecuencia & Porcentaje & Porcentaje válido & $\begin{array}{c}\text { Porcentaje } \\
\text { acumulado }\end{array}$ \\
\hline \multirow{2}{*}{ Válido } & NO & 3 & 23,08 & 23,08 & 23,08 \\
\cline { 2 - 6 } & SI & 10 & 76,92 & 76,92 & 100,0 \\
\hline & Total & 13 & 100,0 & 100,0 & \\
\hline
\end{tabular}

Figura 04

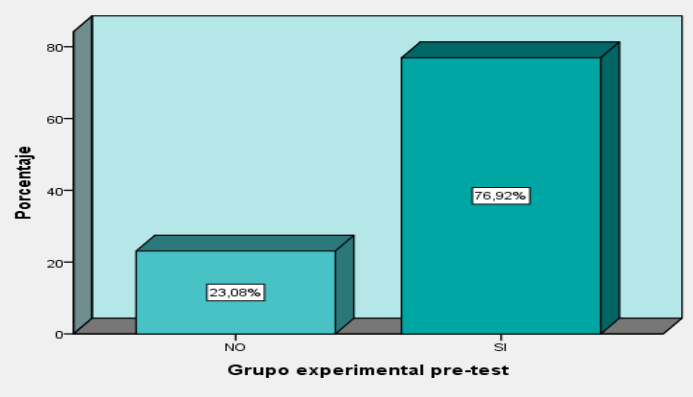

Lista De Cotejo Pre-Test Grupo Control

Variable: Calidad de la expresión oral del idioma inglés DIMENSIÓN: DICCIÓN

Cuadro 15

\begin{tabular}{|l|l|l|l|l|l|}
\hline \multicolumn{2}{|c|}{} & Frecuencia & Porcentaje & Porcentaje válido & $\begin{array}{l}\text { Porcentaje } \\
\text { acumulado }\end{array}$ \\
\hline \multirow{3}{*}{ Válido } & NO & 4 & 33,3 & 33,3 & 33,3 \\
\cline { 2 - 6 } & SI & 8 & 66,67 & 66,67 & 100,0 \\
\cline { 2 - 6 } & Total & 12 & 100,0 & 100,0 & \\
\hline
\end{tabular}


Figura 05

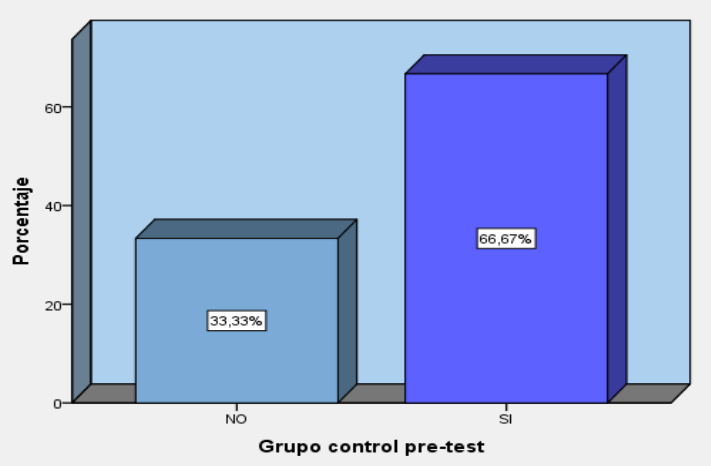

\section{DIMENSIÓN: FLUIDEZ}

\section{Cuadro 16}

\begin{tabular}{llllll} 
& & Frecuencia & Porcentaje & $\begin{array}{l}\text { Porcentaje } \\
\text { válido }\end{array}$ & $\begin{array}{l}\text { Porcentaje } \\
\text { acumulado }\end{array}$ \\
\hline \multirow{2}{*}{ Válido } & NO & 6 & 50,0 & 50,0 & 50,0 \\
\cline { 2 - 6 } & SI & 6 & 50,0 & 50,0 & 100,0 \\
\cline { 2 - 6 } & Total & 12 & 100,0 & 100,0 & \\
\hline
\end{tabular}

Figura 06

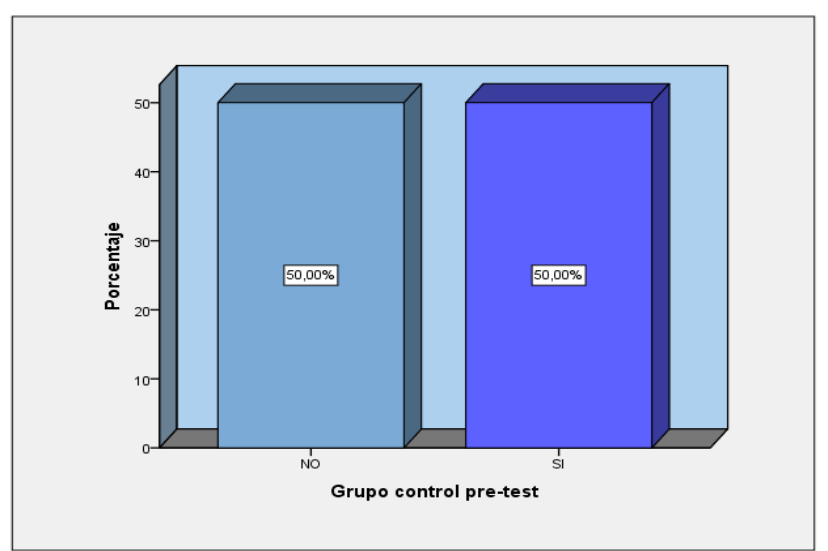

\section{DIMENSIÓN: CLARIDAD}

\section{Cuadro 17}

\begin{tabular}{llllll} 
& & Frecuencia & Porcentaje & $\begin{array}{l}\text { Porcentaje } \\
\text { válido }\end{array}$ & $\begin{array}{l}\text { Porcentaje } \\
\text { acumulado }\end{array}$ \\
\hline \multirow{2}{*}{ Válido } & NO & 6 & 50,0 & 50,0 & 50,0 \\
\cline { 2 - 6 } & SI & 6 & 50,0 & 50,0 & 100,0 \\
\cline { 2 - 6 } & Total & 12 & 100,0 & 100,0 & \\
\hline
\end{tabular}


Figura 07

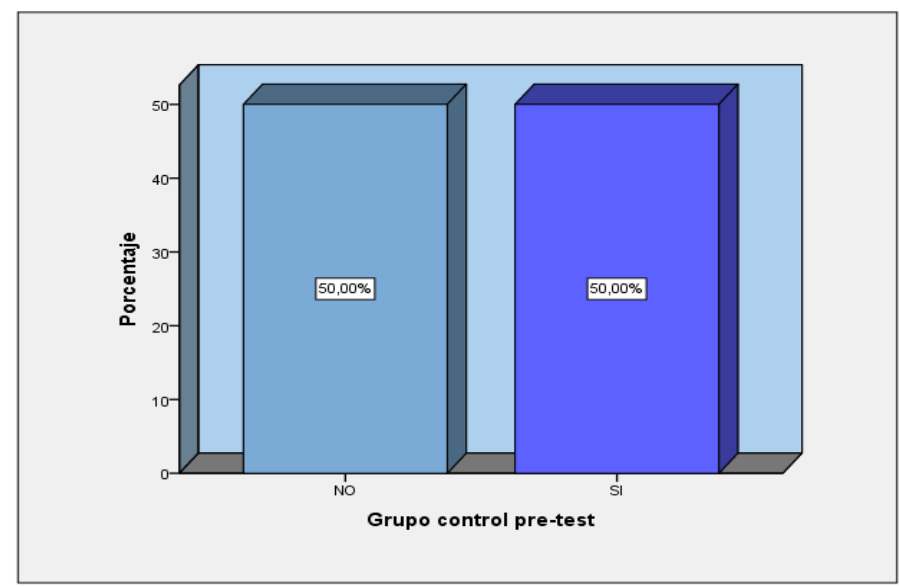

DIMENSIÓN: RITMO

Cuadro 18

\begin{tabular}{lll|lll} 
& & Frecuencia & Porcentaje & $\begin{array}{l}\text { Porcentaje } \\
\text { válido }\end{array}$ & $\begin{array}{l}\text { Porcentaje } \\
\text { acumulado }\end{array}$ \\
\hline \multirow{2}{*}{ Válido } & NO & 9 & $\mathbf{9 5 , 0}$ & $\mathbf{7 5 , 0}$ & $\mathbf{7 5 , 0}$ \\
\cline { 2 - 6 } & SI & $\mathbf{3}$ & $\mathbf{2 5 , 0}$ & $\mathbf{2 5 , 0}$ & $\mathbf{1 0 0 , 0}$ \\
\cline { 2 - 6 } & Total & $\mathbf{1 2}$ & $\mathbf{1 0 0 , 0}$ & $\mathbf{1 0 0 , 0}$ & \\
\hline
\end{tabular}

Figura 08

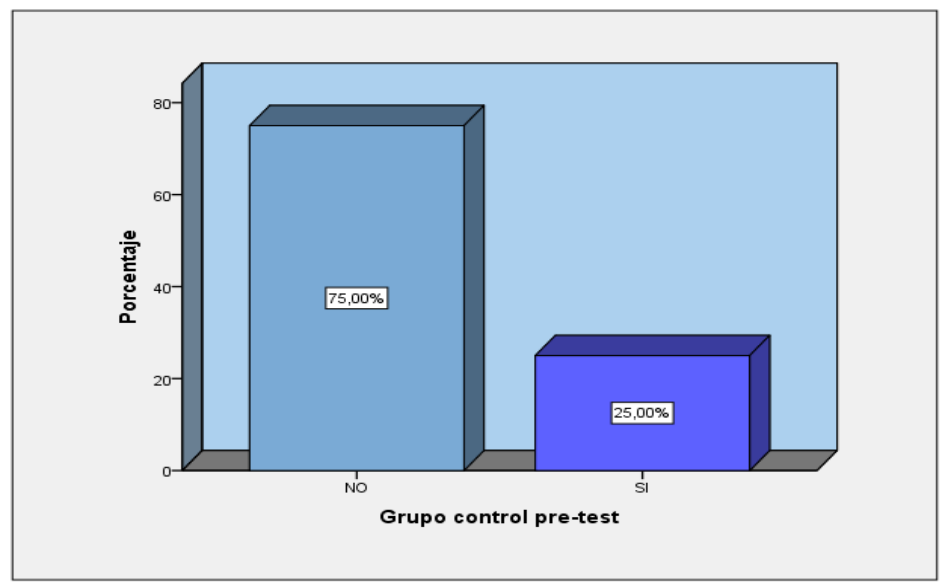

Resultados del Pre-Test

Lista de Cotejo Post-Test Grupo Experimental

Variable: Calidad de la expresión oral del idioma inglés

DIMENSIÓN: DICCIÓN

Cuadro 19

\begin{tabular}{|c|c|c|c|c|}
\hline & Frecuencia & Porcentaje & $\begin{array}{l}\text { Porcentaje } \\
\text { válido }\end{array}$ & $\begin{array}{l}\text { Porcentaje } \\
\text { acumulado }\end{array}$ \\
\hline Válido SI & 13 & 100,0 & 100,0 & 100,0 \\
\hline
\end{tabular}


Journal of the Academy | 126 |

Figura 9

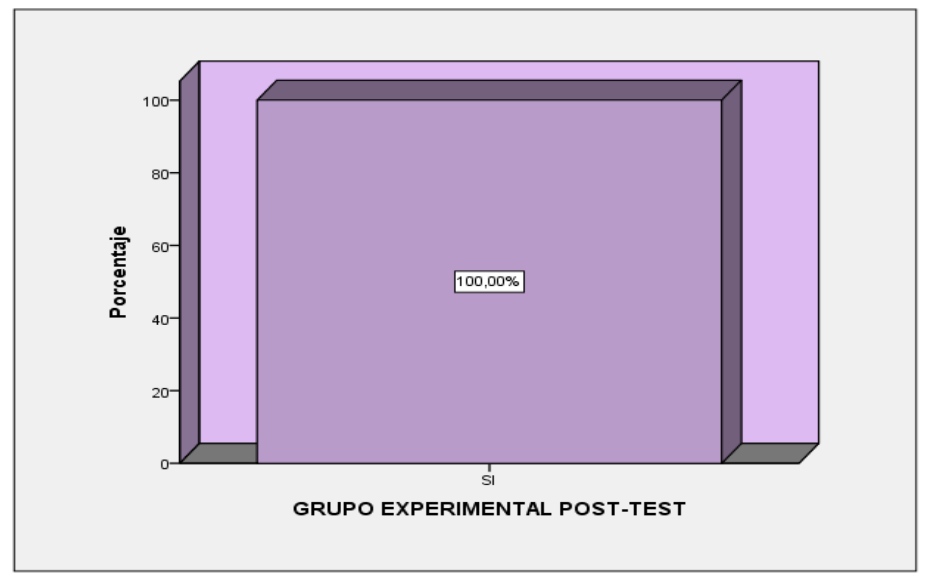

DIMENSIÓN: FLUIDEZ

Cuadro 20

\begin{tabular}{ll|lll} 
& Frecuencia & Porcentaje & $\begin{array}{l}\text { Porcentaje } \\
\text { válido }\end{array}$ & $\begin{array}{l}\text { Porcentaje } \\
\text { acumulado }\end{array}$ \\
\hline Válido SI & 13 & 100,0 & 100,0 & 100,0 \\
\hline
\end{tabular}

Figura 10

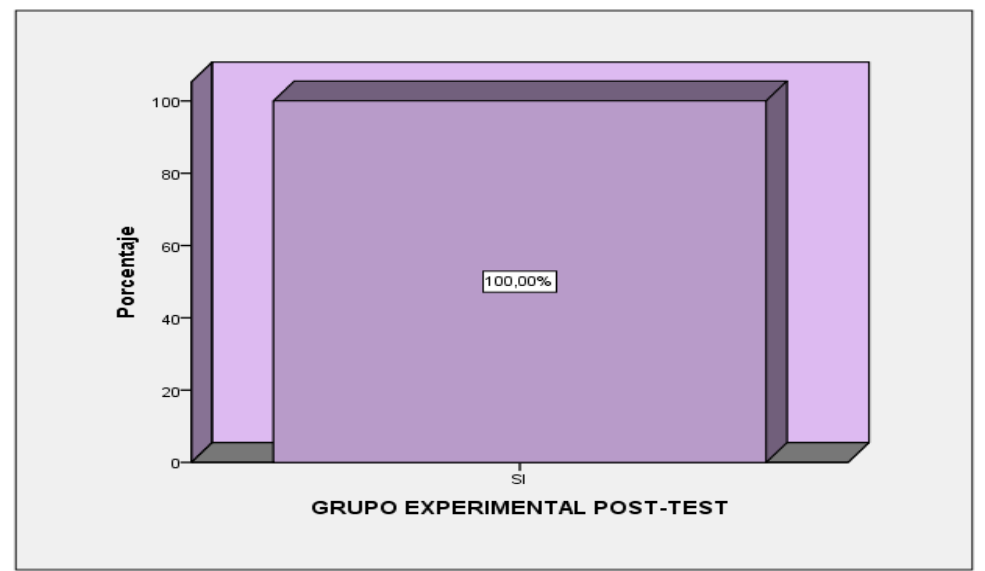

\section{DIMENSIÓN: CLARIDAD}

\section{Cuadro 21}

\begin{tabular}{|c|c|c|c|c|c|}
\hline & & Frecuencia & Porcentaje & $\begin{array}{l}\text { Porcentaje } \\
\text { válido }\end{array}$ & $\begin{array}{l}\text { Porcentaje } \\
\text { acumulado }\end{array}$ \\
\hline & NO & 2 & 15,4 & 15,4 & 15,4 \\
\hline \multirow[t]{2}{*}{ Válido } & SI & 11 & 84,6 & 84,6 & 100,0 \\
\hline & Total & 13 & 100,0 & 100,0 & \\
\hline
\end{tabular}




\section{Figura 11}

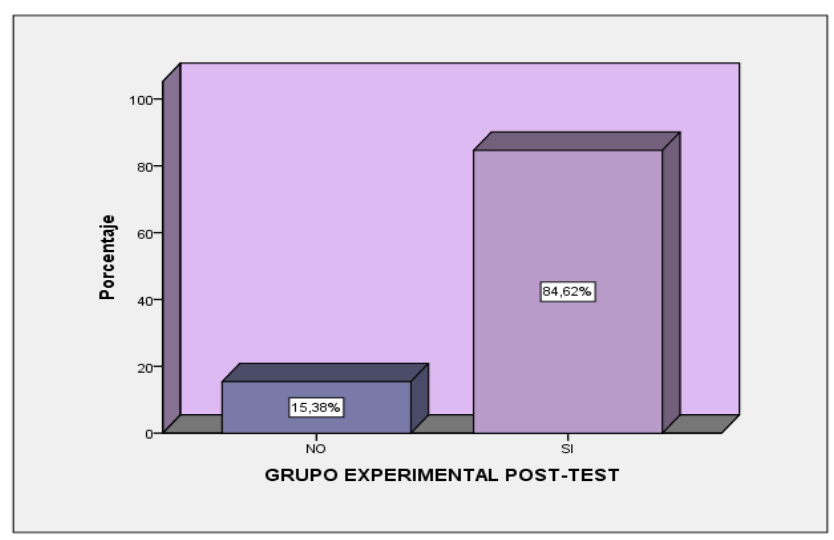

\section{DIMENSION: RITMO}

\section{Cuadro 22}

\begin{tabular}{lll|lll} 
& & Frecuencia & Porcentaje & $\begin{array}{l}\text { Porcentaje } \\
\text { válido }\end{array}$ & $\begin{array}{l}\text { Porcentaje } \\
\text { acumulado }\end{array}$ \\
\hline \multirow{2}{*}{ Válido } & NO & 2 & 15,38 & 15,38 & 15,38 \\
\cline { 2 - 6 } & SI & 11 & 84,62 & $\mathbf{8 4 , 6 2}$ & 100,0 \\
\cline { 2 - 6 } & Total & 13 & 100,0 & 100,0 & \\
\hline
\end{tabular}

\section{Figura 12}

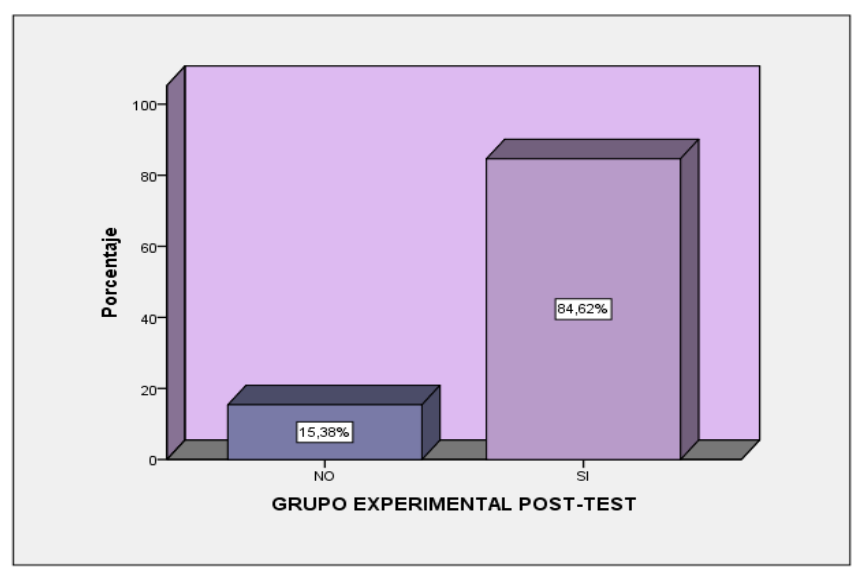

Lista de Cotejo Post-Test Grupo Control

Variable: Calidad de la Expresión Oral del Idioma Inglés DIMENSIÓN: DICCIÓN

\section{Cuadro 23}

\begin{tabular}{|c|c|c|c|c|}
\hline & Frecuencia & Porcentaje & $\begin{array}{l}\text { Porcentaje } \\
\text { válido }\end{array}$ & $\begin{array}{l}\text { Porcentaje } \\
\text { acumulado }\end{array}$ \\
\hline Válido SI & 12 & 100,0 & 100,0 & 100,0 \\
\hline
\end{tabular}




\section{Figura 13}

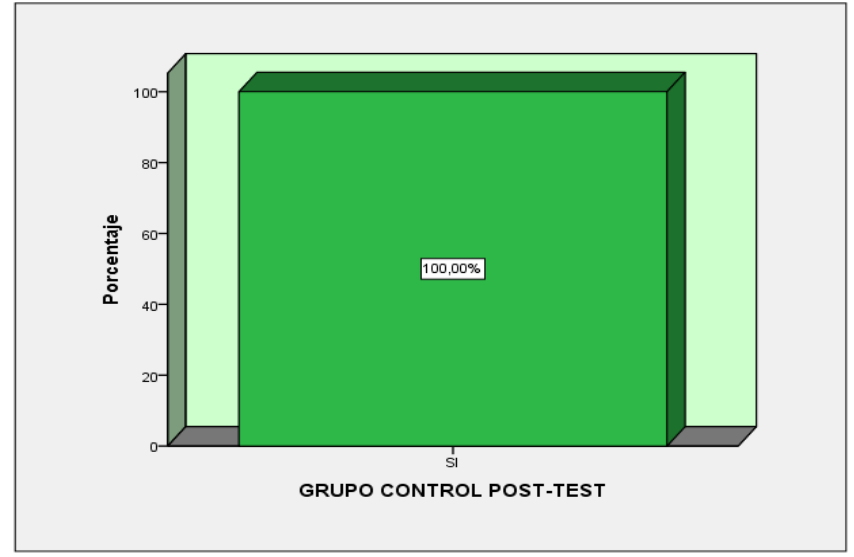

\section{DIMENSIÓN: FLUIDEZ}

Cuadro 24

\begin{tabular}{|c|c|c|c|c|}
\hline & Frecuencia & Porcentaje & $\begin{array}{l}\text { Porcentaje } \\
\text { válido }\end{array}$ & $\begin{array}{l}\text { Porcentaje } \\
\text { acumulado }\end{array}$ \\
\hline $\begin{array}{ll}\text { Válido } & \text { SI } \\
\end{array}$ & 12 & 100,0 & 100,0 & 100,0 \\
\hline
\end{tabular}

Figura 14

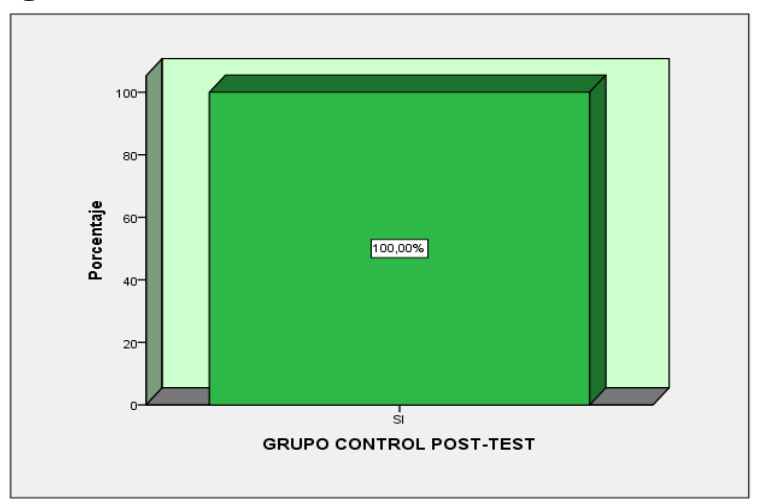

\section{DIMENSIÓN: CLARIDAD}

\section{Cuadro 25}

\begin{tabular}{llllll} 
& & Frecuencia & Porcentaje & Porcentaje válido & $\begin{array}{l}\text { Porcentaje } \\
\text { acumulado }\end{array}$ \\
\hline \multirow{2}{*}{ Válido } & NO & 1 & $\mathbf{8 , 3 3}$ & $\mathbf{8 , 3 3}$ & $\mathbf{8 , 3 3}$ \\
\cline { 2 - 6 } & SI & 11 & 91,67 & 91,67 & 100,0 \\
\cline { 2 - 6 } & Total & 12 & 100,0 & 100,0 & \\
\hline
\end{tabular}




\section{Figura 15}

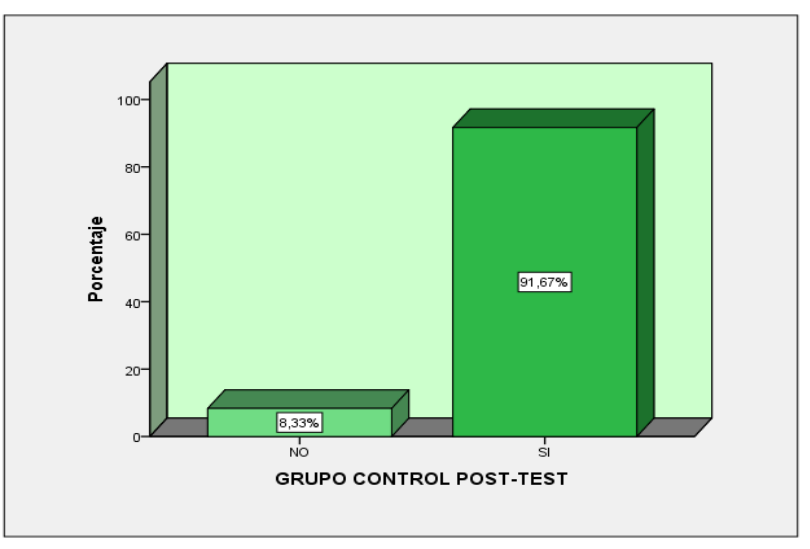

\section{DIMENSIÓN: RITMO}

\section{Cuadro 26}

\begin{tabular}{lll|lll} 
& & Frecuencia & Porcentaje & $\begin{array}{l}\text { Porcentaje } \\
\text { válido }\end{array}$ & $\begin{array}{l}\text { Porcentaje } \\
\text { acumulado }\end{array}$ \\
\hline Válido & NO & 2 & 16,67 & 16,67 & 16,67 \\
\cline { 2 - 6 } & SI & 10 & 83,33 & $\mathbf{8 3 , 3 3}$ & 100,0 \\
\cline { 2 - 6 } & Total & 12 & 100,0 & 100,0 & \\
\hline
\end{tabular}

\section{Figura 16}

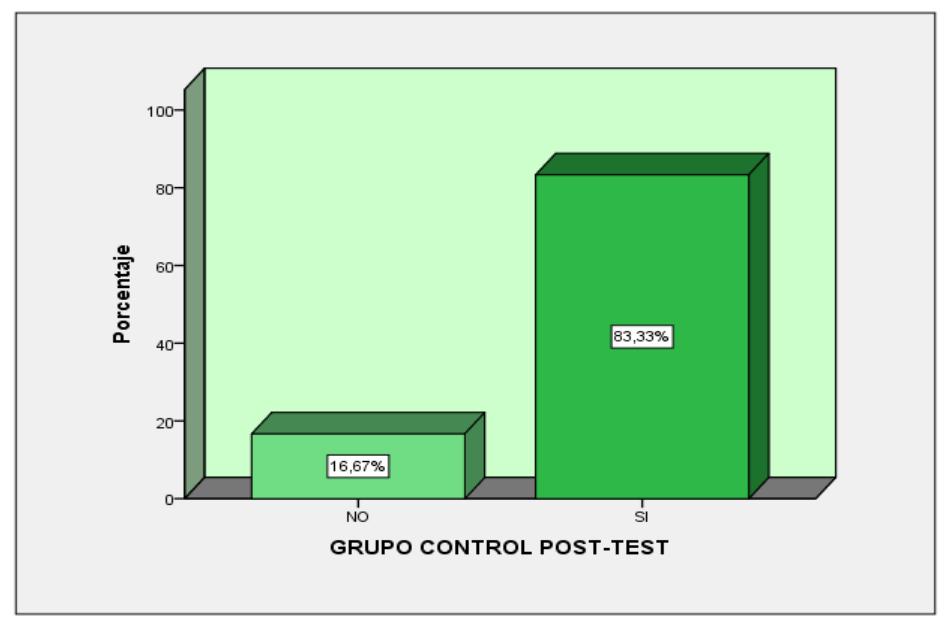

\section{Planteamiento de la Hipótesis General}

HG. La dramatización como herramienta didáctica mejora significativamente la calidad de la expresión oral del idioma inglés en los niños y niñas de 5 años de la institución Educativa Privada Canonesas De la Cruz-Pueblo Libre, Lima-2018.

Ho. La dramatización como herramienta didáctica no mejora significativamente la calidad de la expresión oral del idioma inglés en los niños y niñas de 5 años de la institución Educativa Privada Canonesas De la Cruz-Pueblo Libre, Lima-2018.

Regla de decisión

- $\quad$ Si p valor $=$ Sig. Es $>\boldsymbol{\alpha} \mathbf{0 . 0 5}$, se acepta la hipótesis nula, se rechaza la hipótesis alterna.

- $\quad$ Si p valor $=$ Sig. Es $<\boldsymbol{\alpha} \mathbf{0 . 0 5}$, se rechaza la hipótesis nula, se acepta la hipótesis alterna. 


\section{Journal of the Academy $|130|$}

Estadísticos de la prueba de hipótesis

Diferencia de medias de la variable dependiente: la calidad de la expresión oral del idioma inglés en los niños y niñas de 5 años.

\begin{tabular}{lllll}
\hline & N & Media & Desviación típ. & $\begin{array}{l}\text { Error típ. de la } \\
\text { media }\end{array}$ \\
\hline Pre test & 25 & 5.15 & 1.323 & .162 \\
Post test & 25 & 7.52 & 1.201 & .143 \\
\hline
\end{tabular}

Significancia de la variable dependiente: la calidad de la expresión oral del idioma inglés en los niños y niñas de 5 años.

\begin{tabular}{|c|c|c|c|c|c|c|c|}
\hline & \multicolumn{7}{|c|}{ Valor de prueba $=0$} \\
\hline & \multirow{3}{*}{$\mathrm{t}$} & \multirow{3}{*}{$\mathrm{gl}$} & \multirow{3}{*}{$\begin{array}{l}\text { Sig. } \\
\text { (bilateral) }\end{array}$} & \multirow{3}{*}{$\begin{array}{l}\text { Diferencia } \\
\text { de medias }\end{array}$} & \multirow{2}{*}{\multicolumn{3}{|c|}{$\begin{array}{l}95 \% \text { Intervalo } \\
\text { confianza para } \\
\text { diferencia }\end{array}$}} \\
\hline & & & & & & & \\
\hline & & & & & Inferior & Superior & \\
\hline Pre test & 30.110 & 33 & .000 & 5.742 & 5.16 & 6.09 & \\
\hline Post test & 50.414 & 38 & .000 & 7.620 & 7.12 & 8.08 & \\
\hline
\end{tabular}

Interpretación: Los datos estadísticos confirman las medias conseguidas por el grupo de estudio, respecto a la prueba de entrada aplicada a los los niños y niñas de 5 años alcanzó un promedio de 5.15, luego de la aplicación de la dramatización como herramienta didáctica se expresó en el post test una media de 7.52 puntos.

De manera que esta herramienta pedagógica resultó favorable porque se incrementó en 2.37 puntos. Asimismo, se manifiesta la significancia de .000 menor a lo estipulado 0.05 , por lo cual se acepta la hipótesis alterna. Confirmándose que la dramatización como herramienta didáctica no mejora significativamente la calidad de la expresión oral del idioma inglés en los niños y niñas de 5 años de la institución Educativa Privada Canonesas De la Cruz-Pueblo Libre, Lima-2018.

\section{Planteamiento de hipótesis específicas}

Hipótesis específica 01

H1. El juego de roles mejora significativamente la calidad del aprendizaje del idioma inglés en los niños y niñas de 5 años institución Educativa Privada Canonesas De la Cruz-Pueblo Libre, Lima-2018.

Ho. El juego de roles no mejora significativamente la calidad del aprendizaje del idioma inglés en los niños y niñas de 5 años institución Educativa Privada Canonesas De la Cruz-Pueblo Libre, Lima-2018.

Diferencia de medias de la dimensión el juego de roles

\begin{tabular}{lllll}
\hline & $\mathrm{N}$ & Media & Desviación típ. & $\begin{array}{l}\text { Error típ. de la } \\
\text { media }\end{array}$ \\
\hline Pre test & 25 & 6.20 & 1.313 & .177 \\
\hline Post test & 25 & 7.85 & 1.201 & .141 \\
\hline
\end{tabular}

Significancia de la dimensión el juego de roles

\begin{tabular}{|c|c|c|c|c|c|c|}
\hline \multicolumn{7}{|c|}{ Valor de prueba $=0$} \\
\hline \multirow[t]{3}{*}{$\mathrm{t}$} & \multirow[t]{3}{*}{$\mathrm{gl}$} & \multirow{3}{*}{\multicolumn{2}{|c|}{$\begin{array}{r}\text { Sig. (bilateral)Diferencia } \\
\text { de medias }\end{array}$}} & \multicolumn{3}{|c|}{$95 \% \quad$ Intervalo } \\
\hline & & & & $\begin{array}{l}\text { confianza } \\
\text { diferencia }\end{array}$ & para & \\
\hline & & & & Inferior & Superior & \\
\hline 32.543 & 39 & .000 & 6.200 & 5.04 & 6.02 & \\
\hline 49.883 & 39 & .000 & 7.8563 & 6.12 & 8.08 & \\
\hline
\end{tabular}




\section{Journal of the Academy $|131|$}

Los datos estadísticos ratifican las medias logradas por el grupo de estudio, respecto a la prueba de entrada aplicada a los niños y niñas de 5 años alcanzó un promedio de 6.20 , luego de la aplicación en la dramatización como herramienta didáctica se reflejó en el post test una media de 7.85 puntos.

De manera que esta herramienta didáctica repercutió favorablemente porque se incrementó en 1.65 puntos. Asimismo, se manifiesta la significancia de .000 menor a lo estipulado 0.05 , por lo cual se acepta la hipótesis alterna. Confirmándose que el juego de roles mejora significativamente la calidad del aprendizaje del idioma inglés en los niños y niñas de 5 años institución Educativa Privada Canonesas De la Cruz-Pueblo Libre, Lima-2018.

\section{Hipótesis específica 02}

H2. Las mímicas mejoran significativamente la calidad de la expresión oral del idioma inglés en los niños y niñas de 5 años institución Educativa Privada Canonesas De la Cruz-Pueblo Libre, Lima-2018. Ho. Las mímicas no mejoran significativamente la calidad de la expresión oral del idioma inglés en los niños y niñas de 5 años institución Educativa Privada Canonesas De la Cruz-Pueblo Libre, Lima-2018. Diferencia de medias de la dimensión la mímica

\begin{tabular}{lllll}
\hline & $\mathrm{N}$ & Media & Desviación típ. & $\begin{array}{l}\text { Error típ. de la } \\
\text { media }\end{array}$ \\
\hline Pre test & 25 & 5.87 & 1.515 & .201 \\
\hline Post test & 25 & 7.95 & 1.311 & .151 \\
\hline
\end{tabular}

Significancia de la dimensión la mímica

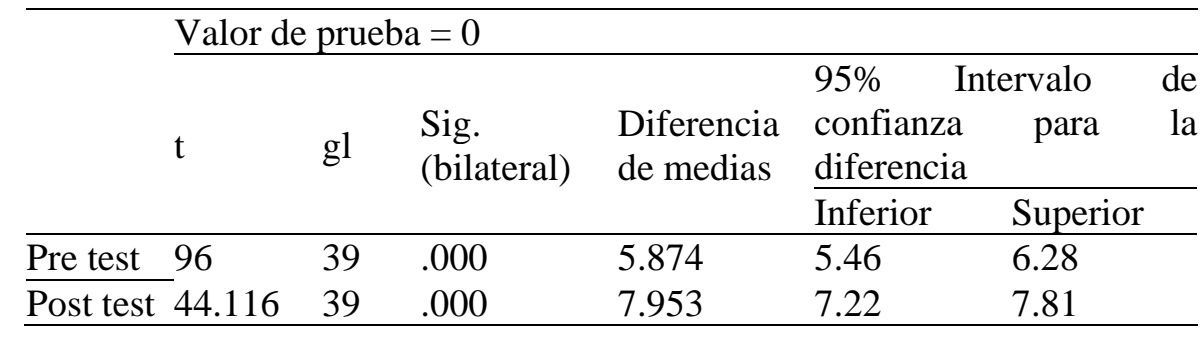

Los datos estadísticos reafirman las medias logradas por el grupo de estudio, respecto a la prueba de entrada aplicada a los niños y niñas de 5 años alcanzó un promedio de 5.87, luego de la aplicación en la dramatización como herramienta didáctica se manifestó en el post test una media de 7.95 puntos. De manera que esta herramienta didáctica repercutió favorablemente porque se incrementó en 2.08 puntos. Asimismo, se manifiesta la significancia de .000 menor a lo estipulado 0.05 , por lo cual se acepta la hipótesis alterna. Confirmándose que la mímica mejora significativamente la calidad de la expresión oral del idioma inglés en los niños y niñas de 5 años institución Educativa Privada Canonesas De la Cruz-Pueblo Libre, Lima-2018.

\section{Hipótesis específica 03}

H3. La improvisación permite una mayor fluidez en la calidad de la expresión oral del idioma inglés en los niños y niñas de 5 años, institución Educativa Privada Canonesas De la Cruz-Pueblo Libre, Lima2018.

Ho. La improvisación no permite una mayor fluidez en la calidad de la expresión oral del idioma inglés en los niños y niñas de 5 años, institución Educativa Privada Canonesas De la Cruz-Pueblo Libre, Lima2018.

Diferencia de medias de la dimensión la improvisación

\begin{tabular}{lllll}
\hline & $\mathrm{N}$ & Media & Desviación típ. & $\begin{array}{l}\text { Error típ. de la } \\
\text { media }\end{array}$ \\
\hline Pre test & 25 & 5.50 & 1.363 & .191 \\
Post test & 25 & 7.94 & 1.424 & .170 \\
\hline
\end{tabular}


Journal of the Academy $|132|$

Significancia de la dimensión la improvisación

\begin{tabular}{|c|c|c|c|c|c|c|}
\hline & \multicolumn{6}{|c|}{ Valor de prueba $=0$} \\
\hline & \multirow[t]{2}{*}{$\mathrm{t}$} & \multirow[t]{2}{*}{$\mathrm{gl}$} & \multirow[t]{2}{*}{ Sig. (bilateral) } & \multirow[t]{2}{*}{$\begin{array}{l}\text { Diferencia } \\
\text { de medias }\end{array}$} & \multicolumn{2}{|c|}{$\begin{array}{l}\text { 95\% Intervalo de } \\
\text { confianza para la } \\
\text { diferencia }\end{array}$} \\
\hline & & & & & Inferior & Superior \\
\hline Pre test & 30.022 & 39 & .000 & 5.500 & 5.41 & 6.19 \\
\hline Post test & 48.758 & 39 & .000 & 7.947 & 6.50 & 9.14 \\
\hline
\end{tabular}

Los datos estadísticos confirman las medias logradas por el grupo de estudio, respecto a la prueba de entrada aplicada a los niños y niñas de 5 años alcanzó un promedio de 5.50, luego de la aplicación en la dramatización como herramienta didáctica se reflejó en el post test una media de 7.94 puntos. De manera que esta herramienta didáctica resultó favorable porque se incrementó en 2.44 puntos. Asimismo, se manifiesta la significancia de .000 menor a lo estipulado 0.05 , por lo cual se acepta la hipótesis alterna. Confirmándose que la improvisación permite una mayor fluidez en la calidad de la expresión oral del idioma inglés en los niños y niñas de 5 años, institución Educativa Privada Canonesas De la Cruz-Pueblo Libre, Lima-2018.

\section{Hipótesis específica 04}

H4. Las dramatizaciones breves mejora significativamente la expresión oral en el idioma inglés en los niños y niñas de 5 años, institución Educativa Privada Canonesas De la Cruz-Pueblo Libre, Lima-2018. Ho. Las dramatizaciones breves no mejoran significativamente la expresión oral en el idioma inglés en los niños y niñas de 5 años, institución Educativa Privada Canonesas De la Cruz-Pueblo Libre, Lima2018.

Diferencia de medias de la dimensión las dramatizaciones breves

\begin{tabular}{lllll}
\hline & $\mathrm{N}$ & Media & Desviación típ. & $\begin{array}{l}\text { Error típ. de la } \\
\text { media }\end{array}$ \\
\hline Pre test & 25 & 5.90 & 1.324 & .175 \\
\hline Post test & 25 & 8.24 & 1.448 & .195 \\
\hline
\end{tabular}

Significancia de la dimensión las dramatizaciones breves

\begin{tabular}{lllllll}
\hline \multicolumn{2}{l}{ Valor de prueba $=0$} \\
\cline { 3 - 6 } & $\mathrm{t}$ & $\mathrm{gl}$ & $\begin{array}{l}\text { Sig. } \\
\text { (bilateral) }\end{array}$ & $\begin{array}{l}\text { Diferencia de } \\
\text { medias }\end{array}$ & $\begin{array}{l}\text { 95\% Intervalo de } \\
\text { diferencia }\end{array}$ \\
\cline { 4 - 7 } & & & & Inferior & Superior \\
\hline Pre test & 30.302 & 39 & .000 & 5.900 & 5.56 & 6.06 \\
\hline Post test & 47.854 & 39 & .000 & 8.246 & 7.89 & 7.98 \\
\hline
\end{tabular}

Los datos estadísticos confirman las medias logradas por el grupo de estudio, respecto a la prueba de entrada aplicada a los niños y niñas de 5 años alcanzó un promedio de 5.90, luego de la aplicación en la dramatización como herramienta didáctica se reflejó en el post test una media de 8.24 puntos. De manera que esta estrategia didáctica resultó favorable porque se incrementó en 2.34 puntos. Asimismo, se manifiesta la significancia de .000 menor a lo estipulado 0.05 , por lo cual se acepta la hipótesis alterna. Confirmándose que las dramatizaciones breves mejora significativamente la expresión oral en el idioma inglés en los niños y niñas de 5 años, institución Educativa Privada Canonesas De la CruzPueblo Libre, Lima-2018. 


\section{CONCLUSIONES}

Los resultados hallados confirman que entre las variables la dramatización como herramienta didáctica y la calidad de la expresión oral del idioma inglés en los niños y niñas de 5 años de la institución Educativa Privada Canonesas De la Cruz-Pueblo Libre, Lima-2018; mejoraron significativamente la expresión oral del idioma inglés en los niños y niñas de 5 años, expresados en la diferencia de medias de 2.37 puntos entre las variables estudiadas. Se determina que la dramatización como herramienta didáctica permite mejorar calidad de la expresión oral del idioma inglés a través del juego de roles, la improvisación, las mímicas y las dramatizaciones breves.

El resultado del análisis de la variable la dramatización como herramienta didáctica y la dimensión el juego de roles permite dar cuenta que aumentaron la expresión oral del idioma inglés, hallándose una diferencia de medias de 1.65. Por lo cual se concluye que la aplicación el juego de roles mejora significativamente la calidad de la expresión oral del idioma inglés en los niños y niñas de 5 años de la institución Educativa Privada Canonesas De la Cruz-Pueblo Libre, haciendo uso de las situaciones cotidianas, los accesorios para los roles y las canciones variadas.

Se confirma que los resultados estadísticos de la variable la dramatización como herramienta didáctica y la dimensión la improvisación mejoran la expresión oral del idioma inglés de los niños y niñas de 5 años, hallándose un incremento de 2.44 puntos entre la variable y la dimensión estudiada. Encontrándose que la improvisación permite una mayor fluidez en la calidad de la expresión oral del idioma inglés en los niños y niñas de 5 años, institución educativa Privada Canonesas De la Cruz-Pueblo Libre, haciendo uso de la acción espontánea y las creaciones propias.

Los resultados estadísticos de la variable la dramatización como herramienta didáctica y la dimensión las mímicas, demostraron la efectividad del uso de la dramatización breves para el incremento de la expresión oral del idioma inglés de los niños y niñas de 5 años, encontrándose un incremento de 2.08 puntos entre la variable y la dimensión estudiada. Por la cual se confirmó que las mímicas mejoran significativamente la calidad de la expresión oral del idioma inglés en los niños y niñas de 5 años institución Educativa Privada Canonesas De la Cruz-Pueblo Libre, haciendo uso del lenguaje no verbal como el cuerpo y el movimiento.

Respecto a la variable la dramatización como herramienta didáctica y la dimensión dramatizaciones breves, se manifestó un incremento de 2.34 puntos en a expresión oral del idioma inglés de los niños y niñas de 5 años con respecto al pre y post test. Concluyendo que las dramatizaciones breves mejora significativamente la expresión oral en el idioma inglés en los niños y niñas de 5 años, institución Educativa Privada Canonesas De la Cruz-Pueblo Libre, a través de los juegos simbólicos, títeres, disfraces y cuentos.

\section{REFERENCIAS BIBLIOGRÁFICAS}

Alabau, I. Y Gil Escudero, G. (1997). Evaluación comparada de la enseñanza y el aprendizaje de la lengua inglesa. España, Francia y Suecia. Madrid: INCE.

Alfaro R., Luis (2009). "La técnica de dramatización y su influencia en la expresión oral de los alumnos de segundo grado de la I.E. "Don José de San Martín" de Tacna". Universidad Privada de Tacna.

Bernardo C., José (2009). Una Didáctica para Hoy. Madrid: Ediciones Rialps, S.A.

Boquete M., G. (2011): El uso del juego dramático en la ensenanza de lenguas: las destrezas orales, Tesis Doctoral, Universidad de Alcalá. 


\section{Journal of the Academy | $134 \mid$}

Camargo, M., Reyes, Y., \& Suárez, D. (2014). El arte en la educación inicial. Obtenido https://www.mineducacion.gov.co/1759/articles- 341880_archivo_pdf_doc_21.pdf

Cardozo C. Margod \& Chicue P. Carlos (2011) "Desarrollo de la expresión oral a través de los títeres en el grado primero A y B de la Institución Educativa Agroecológico Amazónico, Sede John Fitzgerald Kennedy, del Municipio del Paujil, Caquetá" Universidad de La Amazonia Departamento de Educación a Distancia pedagogía infantil Florencia - Caquetá.

Casilla M., Orlando (2009) Tesis “Técnica de dramatización con títeres para mejorar el nivel de práctica de valores en niñas y niños del tercer grado de educación primaria de la I.E. "Mariscal Ramón Castilla" de Ichuña en el año 2007”. Universidad nacional Enrique Guzmán y Valle Lima.

Chen, Z. \& Goh, C. (2011). Teaching oral English in higher education: Challenges to EFL teachers. Teaching in Higher Education, vol. 16, núm. 3, pp. 333-345.

Chiroque, C. (2010) Expresión y comprensión oral. Recuperado el 13 de junio de 2015 de: http://fidokari.es.tl/EXPRESI\%D3N-Y-COMPRENCION-ORAL.htm

Cutillas S.Vicente (2006). "La enseñanza de la dramatización y el teatro: Propuesta didáctica para la enseñanza secundaria. Universidad de Valencia.

Eines, J. y Mantovani, A. (1997): Didáctica de la dramatización, Barcelona: Gedisa.

Figueroa L., Elva (2017) Tesis "Aplicación de la técnica de dramatización para mejorar la expresión oral en el área de inglés en los estudiantes del cuarto grado de educación primaria de la institución educativa Federico Villareal del distrito de Alto Selva Alegre, Arequipa-2016."

Gambini V., Giannina \& Castro R., Medalid (2017) "Influencia del empleo de títeres en el aprendizaje del idioma ingles en los niños de 5 años de la I.E. "Eduardo Ferrick Ring" N 89506, Coishco-Santa, 2014" Universidad Nacional del Santa Facultad de Educación y Humanidades.

Garcés M., Teresa \& Ruiz P. Mery (2010) Tesis: "Diseño y aplicación de obras de títeres que incentiven al cuidado del medio ambiente en las niñas y niños de primer año de Educación Básica de la escuela "María Inés Endara" del barrio Salache Barbapamba del Cantón Salcedo Provincia de Cotopaxi en el periodo 2009-2010" Latacunga- Ecuador. Universidad Técnica de Cotopaxi Unidad Académica de Ciencias Administrativas y Humanísticas Latacunga - Ecuador.

Hernández, S., Fernández, A. Baptista, A. (2014). Metodología de la Investigación. México. Editorial Mc Graw Hill.

Manzano, D. (2014). Porque debe aprender inglés. 5 Buenas razones para motivarle.

Mejía M., Elías (2005); Metodología de la Investigación Científica. Centro de Producción Editorial e Imprenta de la UNMSM.

Mejia, Elías (2005); Técnicas e instrumentos de investigación científica. Centro de Producción Editorial e Imprenta de la UNMSM.

Motos, Tomás (1992) «Las técnicas dramáticas: Conceptualización». Universidad de Valencia.

Navarro S., M. (2006/2007): "Drama, creatividad y aprendizaje vivencial: algunas aportaciones del drama a la educacion emocional", Cuestiones Pedagogicas, 18, 2006/2007.

Núñez C., Luis Y Navarro S., María “Dramatización y Educación aspectos teóricos. 


\section{Journal of the Academy $|135|$}

Programa Curricular De Educacion Inicial (2016) Ministerio De Educación Documento de trabajo elaborado por la Dirección General de Educación Básica Regular.

Shapiama S., Julissa. \& Trigoso A., Shirley (2013) Tesis " Efectos de un Programa de Talleres de Títeres en el Desarrollo de la expresión oral en niños de 4 años de la I.E.I. № 657 'Niños del saber', distrito de Punchana - 2013" Universidad Nacional de la Amazonia Peruana Facultad de Ciencias de la Educación y Humanidades.

Tafur P., Raúl (1995). La Tesis Universitaria. La tesis doctoral, la tesis de maestría, el informe y la monografía. Primera Edición. Editorial Mantaro. Lima, Perú. 432 pp.

Torres, L. (2017). La dramatización como estrategia pedagógica basado en el enfoque colaborativo para mejorar la expresión oral en niños de 4 años de la institución educativa inicial $\mathrm{N}^{\circ} 026$ de Huallanca en el año 2016. Obtenido de Universidad Católica Los Ángeles

Vez, J.M., Martínez, E. Y Lorenzo, A. (2012). La exposición y uso ambiental de la lengua extranjera en contextos no formales mejora su comprensión oral. En MECD.

Villegas, Cenai (2004). "El Uso del Títere en el Desarrollo de la Expresión Oral y Escrita (Escuela Básica Nacional José Antonio Calcaño)”. Universidad Nacional Abierta - Venezuela.

https://www.portaleducativo.net/cuarto-basico/648/Dramatizacion-y-obra-de-teatro

https://es.wikipedia.org/wiki/Idioma_ing1\%C3\%A9s

https://gredos.usal.es/jspui/bitstream/10366/71846/1/Dramatizacion_y_educacion_aspectos_teori.pdf

http://idiomas.ens.uabc.mx/plurilinkgua/docs/v4/1/Carolinaunestudio.pdf

https://www.unir.net/educacion/revista/noticias/la-expresion-oral-en-el-aula-de-ingles-de-

primaria/549201736459/

http://www.ugr.es/ portalin/articulos/pl_numero22/18\%20\%20gabino\%20boquete.pdf

http://institucional.us.es/revistas/cuestiones/18/10\%20drama,\%20creatividad.pdf

Http://Repositorio.Uladech.Edu.Pe/Bitstream/Handle/123456789/3664/DramaTizacion_Expresion_To rres_Ibarra_Lina_Marina.Pdf?Sequence=1\&isAllowed=y 